\title{
Model studies of flow parameters in the area of bridge supports
}

\author{
Gennady Gladkov ${ }^{1, *}$, Konstantin Morgunov ${ }^{1}$, and Yuri Ivanovsky ${ }^{1}$ \\ ${ }^{1}$ Admiral Makarov State University of Maritime and Inland Shipping, 5/7, Dvinskaya str., 198035, \\ Saint-Petersburg, Russia
}

\begin{abstract}
The results of laboratory modeling of the influence of the bridge crossing supports erected during the construction of the highway near the existing railway bridge on the flow characteristics in the channel of the Neva River are presented. Modeling was carried out for two options for the location of the new bridge supports relative to the existing bridge structures. The limits of changes in the characteristics of the river flow are taken into account - the maximum, minimum and residual flows and benchmarks of the water level in the channel. Studies have shown that the construction of the designed bridge supports in the channel does not cause significant changes in the flow structure. There is a redistribution of the flow rate in the sections of the existing and designed bridges. The average velocities in the navigable span of the existing bridge are somewhat reduced when new supports are built. The velocity diagram is aligned along the width of the central and side spans, and a vortex wake is more clearly formed in the area behind the supports.
\end{abstract}

\section{Introduction}

The paper presents the materials of laboratory studies of the influence of supports erected in the channel during the construction of a bridge over the Neva River as part of the design of a latitudinal highway of high-speed traffic on the characteristics of the river flow.

Bridge crossings erected in the channels of navigable rivers have an impact on the kinematics of the flow and create certain restrictions for navigation: they can cause a change in the level and speed structure of the flow, lead to the appearance of dump currents and undesirable deformations of the river bottom. These factors lead to a change in the controllability conditions of the ship when passing under the bridge span and to an increase in the likelihood of emergencies.

In the course of many years of practice in the design, construction and operation of bridges on navigable rivers, a certain experience has been formed in ensuring navigable conditions in the area of bridge crossings. For the computational substantiation of course arrangements, it is customary to use materials from field and laboratory studies, during which it is possible to assess the effect of the bridge crossing on the hydraulic characteristics of the flow and channel.

\footnotetext{
* Corresponding author: vgl@gumrf.ru
} 


\section{Materials and Methods}

In the practice of computational substantiation of engineering measures on rivers, the most difficult tasks to date are modeling the kinematic characteristics of water flow around structures in the river channel and forecasting channel transformations (bottom deformations) in rivers. Such forecasts are of practical interest in the operation of infrastructure of inland waterways, ensuring the stability of navigable passages on free rivers and in the downstream of hydroengineering complexes, as well as in the design and operation of bridge crossings, river training facilities in the channels of navigable rivers and berthing facilities on waterways, etc.

Traditional research methods in the field of channel process [1-5] are based on the use of the geomorphological approach, as well as on the use of the results of hydraulic and hydromorphological studies. The tools of mathematical modeling are widely used in domestic and foreign practice for modeling channel reformation $[6,7]$.

Hydraulic calculations of the characteristics of water movement and bottom reformation in rivers are performed by solving the system of Saint-Venant equations using numerical methods. This system includes the equation of unsteady uneven movement of water in the river, as well as equations of continuity and deformation. The system of equations is closed by specifying two additional functions: the formula for the Chezy coefficient and the formula for the sediment discharge at given initial and boundary conditions, which characterize the hydraulic, morphometric and hydrological data.

The main recommendations for constructing an algorithm for hydraulic calculations are obtained from the results of the studies performed in the works of the authors [8-10].

This paper presents the materials of laboratory studies of the influence of channel supports erected in the channel during the construction of a bridge over the Neva River as part of the design of a latitudinal highway on the characteristics of the river flow. The work was performed in the channel flume of the hydrotechnical laboratory of the Admiral Makarov State University of Maritime and Inland Shipping [11].

The physical picture of the water flow, illustrating the kinematic structure of the flow and local reshaping of the bottom when flowing around the supports, was studied in detail in [12-15]. However, in this case, in order to develop recommendations for ensuring the safety of navigation when passing the navigable span of a movable bridge, taking into account the influence of the designed bridge supports, it was necessary to study the characteristics of the water flow not only near the channel support of the bridge, but also on the navigable passage, including on section of the approach to the section line of the bridge crossing.

When developing approaches to hydraulic modeling, one proceeds from an assessment of physical factors that determine the course of the processes under study, as well as the capabilities of laboratory facilities. On the basis of such estimates using the methods of the similarity theory, the scale of modeling and characteristics of the model are determined [3].

Similarity criteria in hydraulic modeling $[16,17]$ are determined by transforming the Navier-Stokes equations describing the motion of a real (viscous) fluid in nature and on a model. Such parameters are the Froude (Fr), Reynolds (Re) and Euler (Eu) criteria. When modeling open flows, the Euler criterion turns out to be zero $(\mathrm{Eu}=0)$ both for nature and for the model. The presence of a self-similarity zone in terms of the Reynolds number, which is provided when the horizontal and vertical geometric scales are equal, makes it possible to exclude the Reynolds number from the criteria (in case of approximate modeling).

Based on the capabilities of the laboratory setup, as well as the desire to minimize the geometric dimensions of the bridge supports and the navigable span, the need to exclude the effect of the flume walls on the hydraulic structure of the model flow and the influence 
of measuring devices on the velocity structure of the flow, a scale of 1:100 was adopted in the modeling.

Equality of the Froude number for the model and nature leads to the following scales for different values describing the flow parameters:

- geometric similarity is ensured by the equality of all linear scales (length, width and depth) $a_{l}=a_{b}=a_{h}=100$;

- for horizontal and vertical components of flow velocity $a_{v_{\mathrm{h}}}=a_{v_{\mathrm{v}}}=\sqrt{a_{h}}=10$;

- for water consumption $a_{Q}=a_{h}^{5 / 2}=100000$;

- for time $a_{t}=\frac{a_{h}}{a_{v}}=\sqrt{a_{h}}=10$.

On a spatial setup, the following was reproduced on a scale (Fig. 1):

- a fragment of a section of the Neva river flow, including a navigable span and half of adjacent spans;

- conditionally erosion-resistant bottom of the river flow;

- bridge supports of the existing railway bridge;

- bridge supports of the designed automobile bridge.

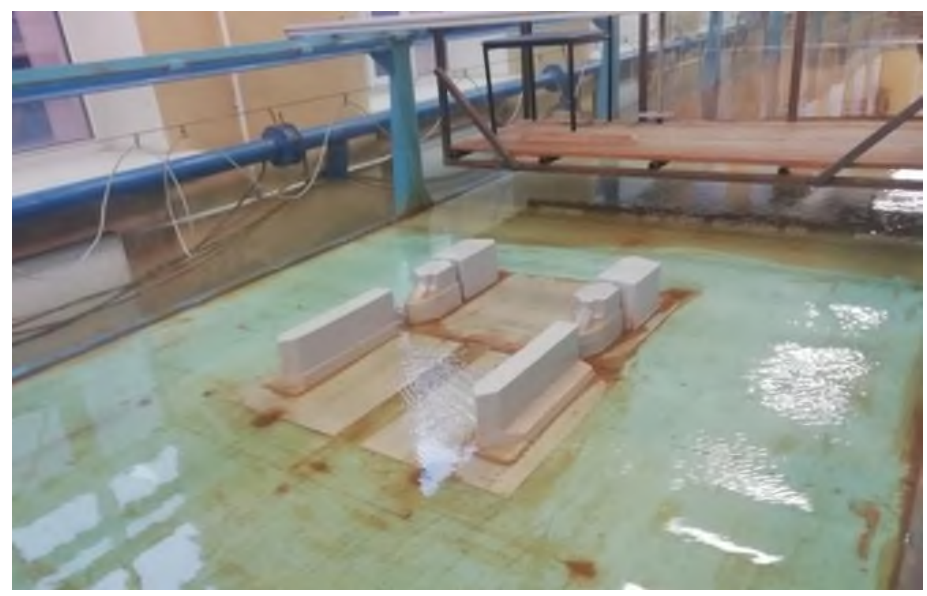

Fig. 1. The location of the supports of the existing and designed bridges in the hydraulic flume.

The assessment of the boundaries of changes in flow parameters (water levels and flow rates in the alignment of the designed bridge), at which the velocity fields in the bridge spans are determined, was carried out on the basis of field measurements at stationary stations located on the Neva River: Novosaratovka village located $11.2 \mathrm{~km}$ upstream of the designed bridge, and Mining University located $13.0 \mathrm{~km}$ downstream.

This estimate gives the following values:

- the maximum water level is determined in accordance with the Rules for navigation of ships when opening St. Petersburg bridges: bridges are not opened when the water level is $150 \mathrm{~cm}$ above the zero water level for the Palace Bridge, for the location of the designed bridge, it is $162 \mathrm{~cm} \mathrm{BS}$;

- the minimum water level is determined by the List of hazardous hydrometeorological phenomena and their criteria for St. Petersburg - in the site section of the designed bridge it is minus $72 \mathrm{~cm} \mathrm{BS}$;

- as the maximum, water flow rates of 30 to $1 \%$ are taken $-3400-4500 \mathrm{~m}^{3} / \mathrm{s}$;

- as the minimum, the average annual water flow rate is taken, which for the Novosaratovka gauging station is $2500 \mathrm{~m}^{3} / \mathrm{s}$. 
In addition, to calibrate the model setup and compare laboratory test data with field measurements, another benchmark was adopted in the bridge site section - $0.125 \mathrm{~m} \mathrm{BS}$. This is the benchmark at which full-scale measurements were performed.

The research methodology involved the execution of several cycles of experiments:

- investigation of the flow structure when flowing around the supports of the existing bridge - everyday (undisturbed) state (Fig. 2,a);

- study of the flow structure at two positions of installation of the supports of the designed bridge - at a distance of $55.0 \mathrm{~m}$ between them (the first design state - Fig. 2, $b$ ) and at a distance of $50.0 \mathrm{~m}$ between them (the second design state - Fig. 2, c).

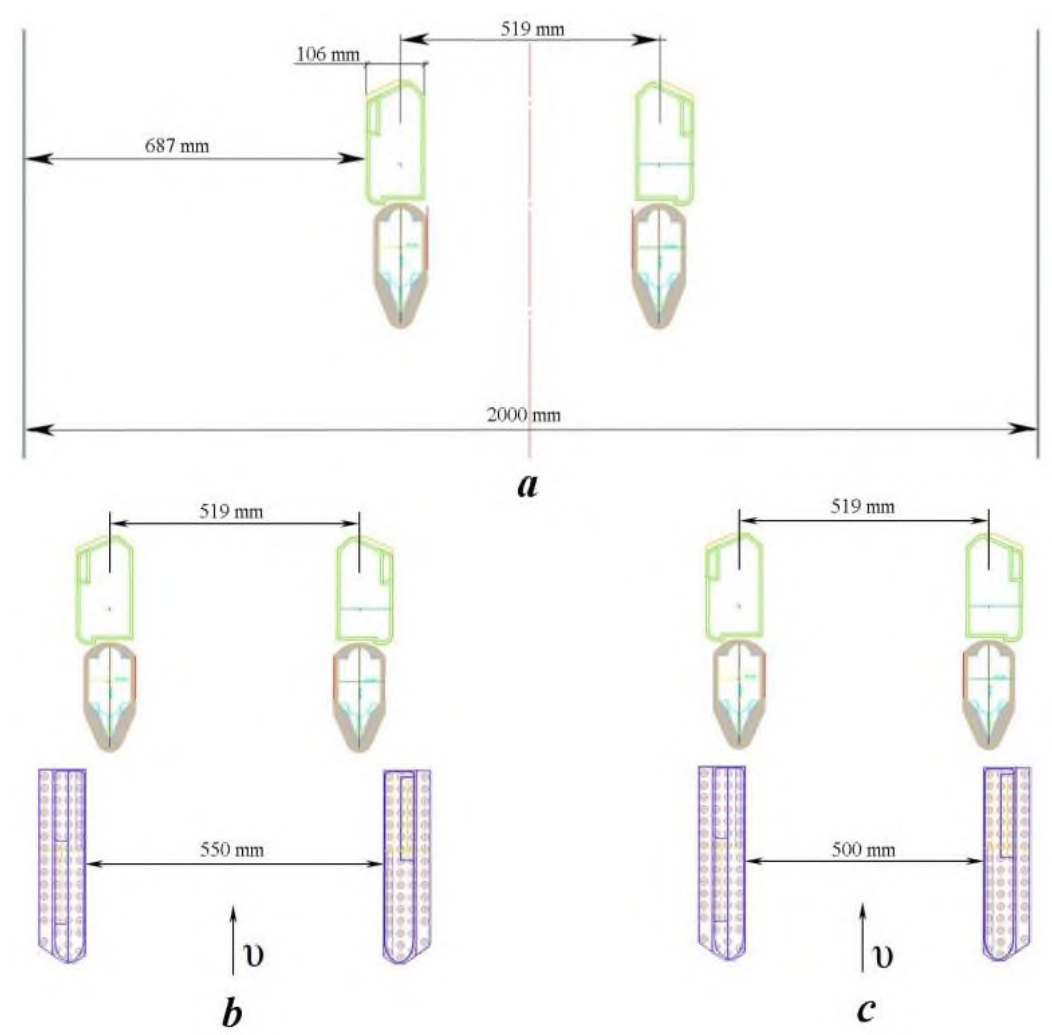

Fig. 2. Location of bridge support models in the flume: $a$ - supports of the existing bridge; $b$ - the first design state; $\mathrm{c}$ - second design state.

The following kinematic characteristics of the flow were measured during each cycle:

- benchmarks of the free surface of water and, consequently, the slopes of the water surface;

- directions, trajectories and velocities of surface currents (measurements were carried out by photographing the movement of specially made multi-colored reflective surface floats with a Canon 7D camera);

- velocities of the currents at different points of the flow cross-section, distributed over the width and depth of the flow (measurements were carried out with a laboratory microspinner).

The measurements were carried out in accordance with a grid of 10 hydraulic wells located along the river flow above and below the bridge (Fig. 3). 


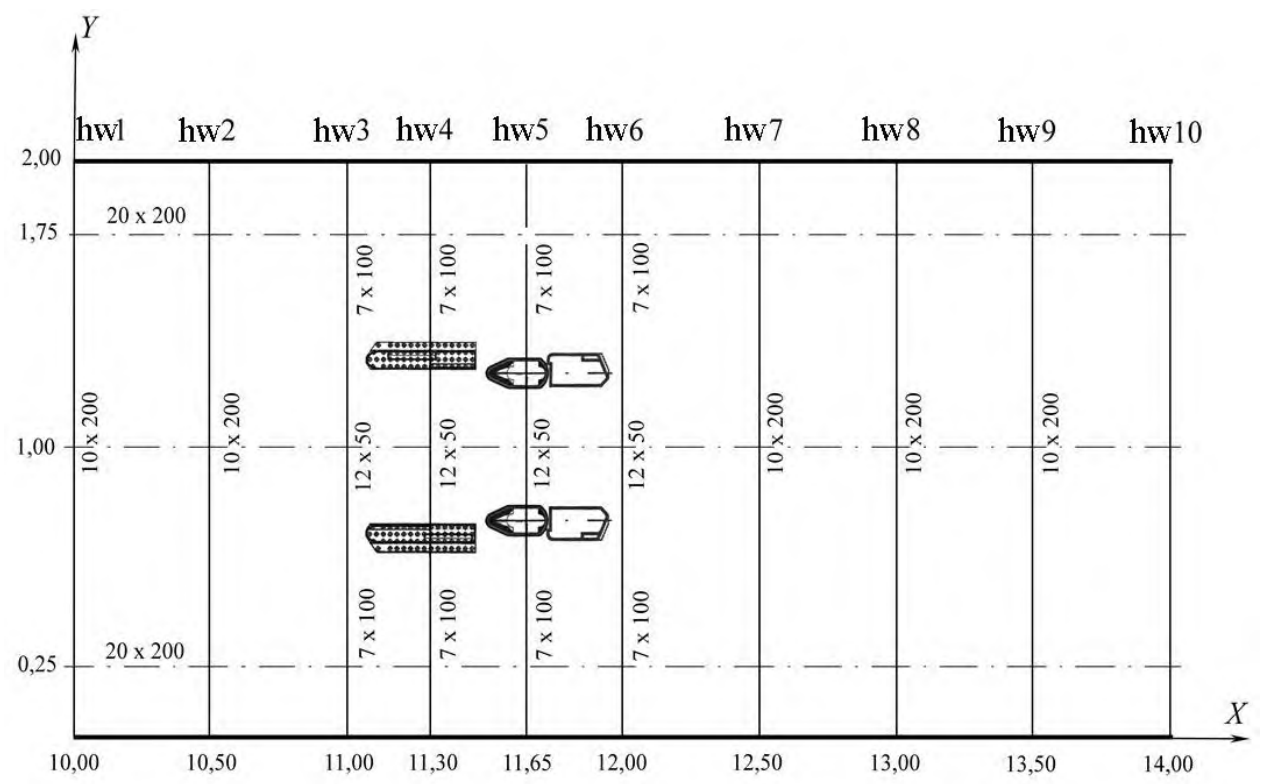

Fig. 3. Grid of measurements in the flume.

In hydraulic modeling on a laboratory setup, a $200 \mathrm{~m}$ wide section of the river flow was adopted, which included a navigable span and two halves of adjacent spans. This required determining the flow rates of water passing through this allocated area. Analysis of the data of field measurements carried out in the water area of the Neva River showed that $60.4 \%$ of the total river flow passes through a section $200 \mathrm{~m}$ wide. Then, taking into account the boundary conditions described above, the following expenses were taken during laboratory tests.

Table 1. River water consumption.

\begin{tabular}{|l|c|c|c|}
\hline River water consumption, $\mathbf{~ m}^{\mathbf{3}} / \mathbf{s}$ & 4500 & 3400 & 2550 \\
\hline $\begin{array}{l}\text { Water consumption in a strip of } \mathbf{2 0 0} \mathbf{~ m} \\
(\mathbf{6 0 . 4 \%}) \mathbf{~} \mathbf{~ m}^{\mathbf{3}} \mathbf{s}\end{array}$ & 2718.0 & 2053.6 & 1540.4 \\
\hline Water consumption in a model, $\mathbf{~} / \mathbf{s}$ & 27.180 & 20.536 & 15.404 \\
\hline
\end{tabular}

The measurements were carried out with a step of $0.1 \mathrm{~m}$ across the flow, between the bridge supports the step of measurements was $0.05 \mathrm{~m}$. Measurements of the average velocity were carried out at a benchmark of 0.6 of the flow depth at all points, except for points located on the longitudinal axis of the flow (on the ship's course). Detailed measurements of velocities in depth were carried out on the ship's course. Measurements were carried out at depths of $0.2 h ; 0.4 h ; 0.6 h ; 0.8 h$, and directly at the bottom.

\section{Results}

The main task of hydraulic studies of a model of a bridge crossing with installed supports of the designed bridge was to determine the effect of newly placed structures on the nature of the distribution of water velocities in the considered section of the river flow.

Measurements of the velocity fields on the model in a common state (only the supports of the existing bridge) were carried out in order to check the adequacy of the model (calibration) and subsequent comparison with the distribution of velocities when installing the supports of the new bridge. 
Of the characteristic features of the flow in a common state, the following can be noted.

1. On the approach to the bridge supports (hydraulic wells from No. 1 to No. 4), the distribution of velocities in the cross section of the flow is rather uniform for all the studied flow rates and levels of surface elevations (Fig. 4, a), while the absolute values of velocities at the same elevation of the surface increase with rising consumptions, which is natural.

2. The distribution of velocities in the site section of the bridge supports (hydraulic well No. 5) is also fairly uniform for all studied cases (Fig. 4, $b$ ).

3. In the site section located directly behind the supports (hydraulic well No. 6), in the areas in the "shadow" of the supports (immediately behind them), vortices appear, the longitudinal velocity components significantly decrease up to the appearance of reverse flows (Fig. 4, $c$ ).

4. The influence of the supports extends far enough downstream. In all the studied hydraulic wells (from No. 7 to No. 10), depressions in the "shadows" of the supports were observed on the velocity plots, decreasing in absolute value downstream.

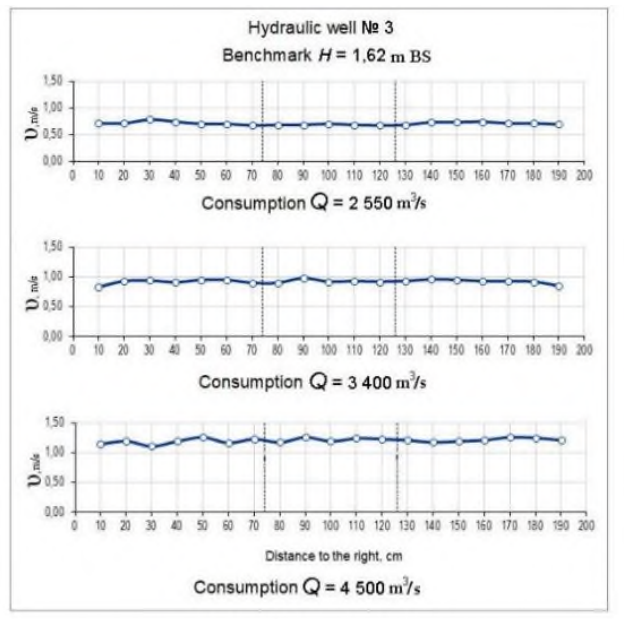

$a$
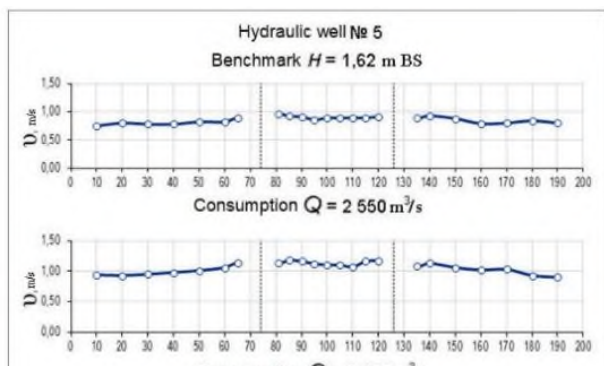
Consumption $Q=3400 \mathrm{~m}^{3} / \mathrm{s}$

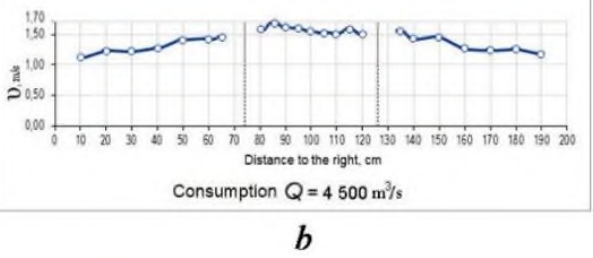

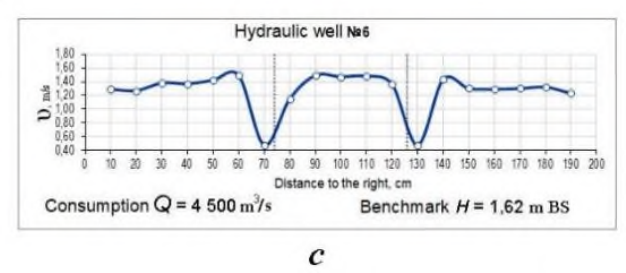

Fig. 4. Distribution of average velocities on hydraulic wells.

5. By depth on the ship's course, the velocities were measured at depths of $0.2 h ; 0.4 h$; $0.6 h ; 0.8 h$ (the depth is measured from the free surface of the flow) and directly at the bottom. In depth, the velocities decrease from surface to bottom (Fig. 5). The distribution of velocities along the flow indicates that the velocities increase in the bridge site section, the diagram is leveled in depth. This is caused by a decrease in the area of the free flow area when it is constrained by the bridge supports. Further, already in hydraulic wells No. 7 No. 8 , the velocities return to the values and distribution characteristic of the first hydraulic wells. 


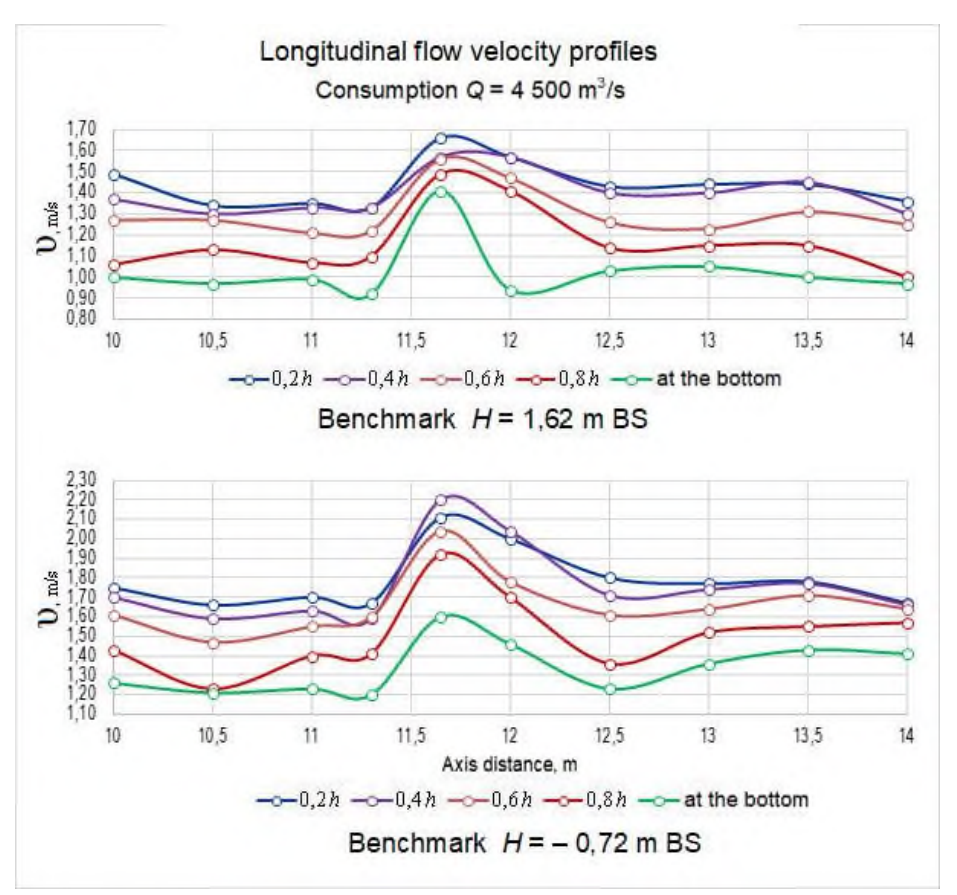

Fig. 5. Velocity distribution along the ship's course throughout the entire section between hydraulic wells No. 1 - No. 10.

Placing the designed supports in the channel of the flume leads to the following changes in the flow.

Contraction of the flow, a decrease in the area of its free cross-section begins earlier than in the common state, upstream, this leads to a redistribution of the flow rate along the flow width.

In a common state (Fig. 6), the average velocity in the central span between the existing supports is $v_{\mathrm{av}}=1.03 \mathrm{~m} / \mathrm{s}$, the average velocity in the lateral spans is $v_{\mathrm{av}}=0.91 \mathrm{~m} / \mathrm{s}$. After the installation of new supports, in the first design state, the average velocity in the navigable span becomes equal to $v_{\mathrm{av}}=0.99 \mathrm{~m} / \mathrm{s}$ (decreased), the average velocity in the side spans also became less $-v_{a v}=0.85 \mathrm{~m} / \mathrm{s}$. And for the second design state, the situation almost did not change in comparison with the common one - the average velocity in the central span is $v_{\mathrm{av}}=1.03 \mathrm{~m} / \mathrm{s}$, the average velocity in the lateral spans is $v_{\mathrm{av}}=0.91 \mathrm{~m} / \mathrm{s}$. Apparently, this occurs due to the reshaping of the velocity diagram. From Fig. 6 it can be seen that the velocities in the lateral spans have leveled off in width. In addition, when additional walls are installed in the flow, the pressure losses caused by the flow around additional obstacles increase, which leads to a certain decrease in velocities. 


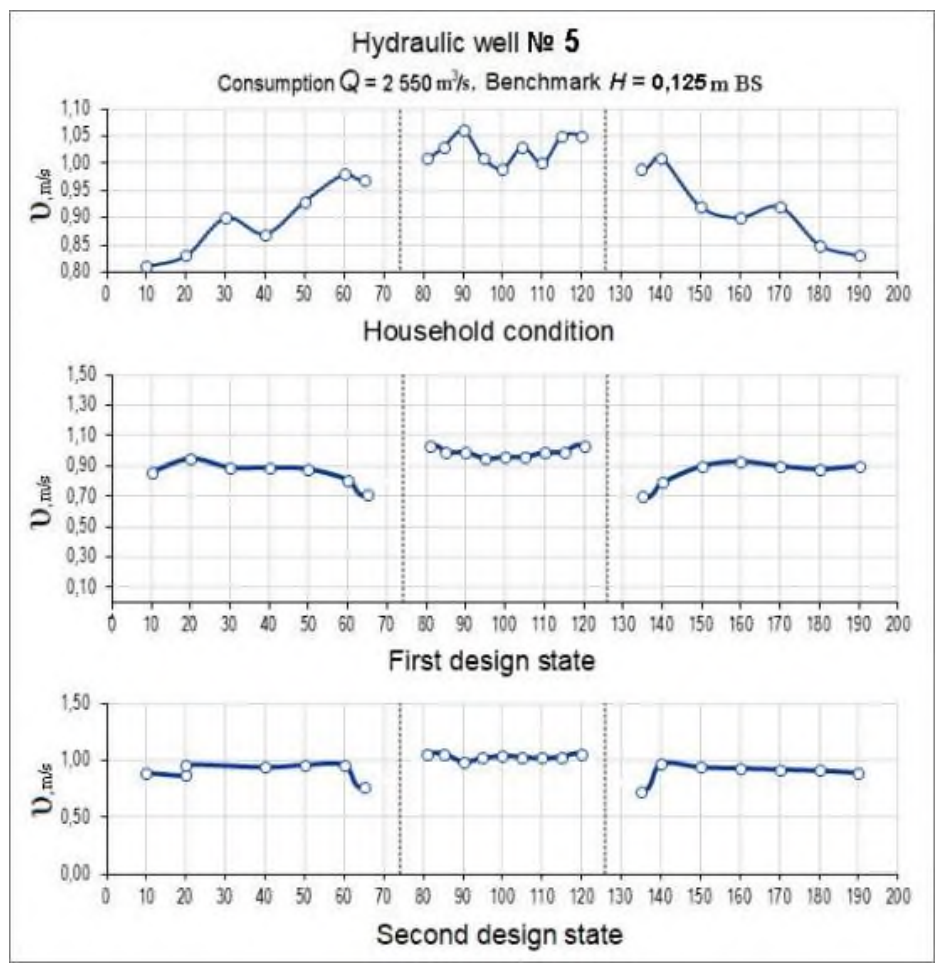

Fig. 6. Distribution of velocities in the hydraulic well No. 5 for household (common) and design states at $Q=2550 \mathrm{~m}^{3} / \mathrm{s}$ and $H=0.125 \mathrm{~m} \mathrm{BS}$.

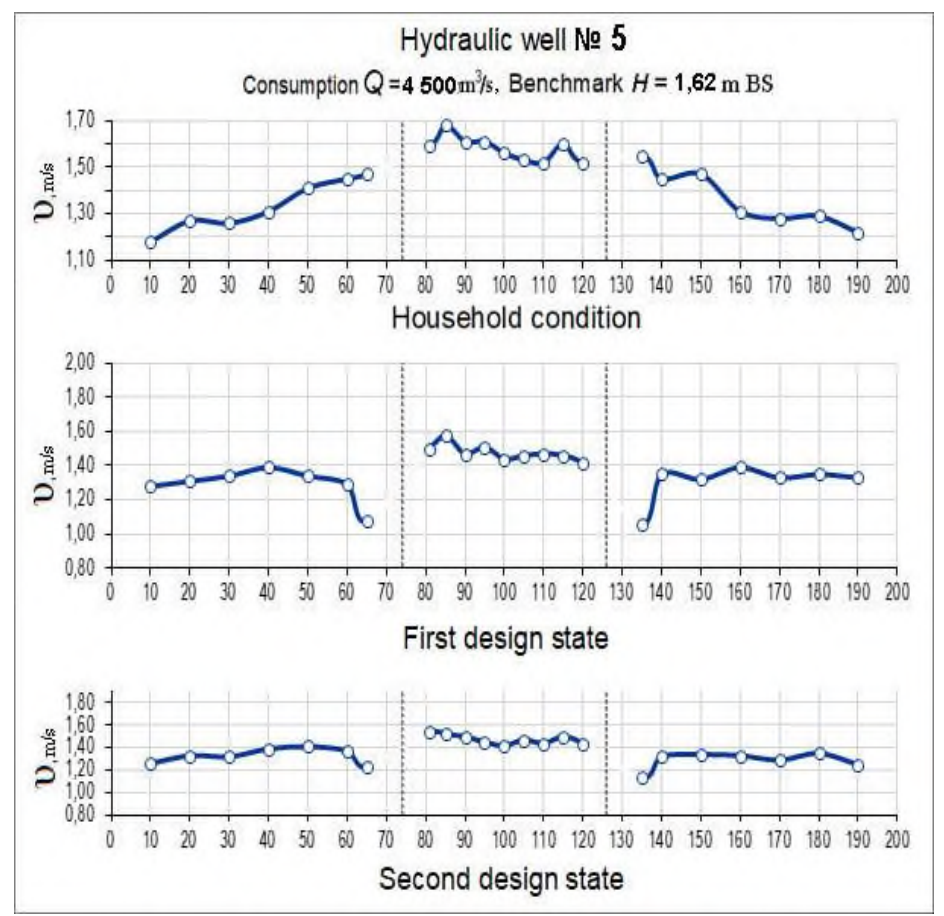

Fig. 7. Distribution of velocities in the hydraulic well No. 5 for household (common) and design states at $Q=4500 \mathrm{~m}^{3} / \mathrm{s}$ and $H=1.62 \mathrm{~m} \mathrm{BS}$. 
As the flow rate increases, the velocity diagrams in the lateral spans change in the design flow states (Fig. 7). In the first design state, the average velocity in the central span increased by $1.76 \%$, in the side spans it decreased by $2.0 \%$. In the second design state, the average velocity in the central span increased by $0.9 \%$, in the side spans - increased by $1.0 \%$.

Downstream, starting from hydraulic well No. 7, changes in the distribution of velocities in cross-sections during the transition from the common state to the design state are very insignificant.

\section{Discussion}

The distribution of speeds along the course of a ship along the flow shows that during the transition from the common state to the design state, the velocity changes along the flow are smoothed out and become gentler (Fig. 8). Installation of additional guide walls (supports of the designed bridge) along the movement of the flow straightens the axis of the central stream of the flow, also causing additional head losses, which leads to a certain decrease in the maximum velocities on the ship's course.

The general picture of the distribution of velocities is presented in the form of a distribution field in Fig. 9. It can be seen that with the construction of new bridge supports, part of the flow is displaced from the central span to the side spans of the bridge, in which the velocities equalize along the width of the spans. In the lower hydraulic wells (No. 7 No. 10), the average velocities decrease somewhat, and a turbulent vortex wake forms behind the bridge supports.

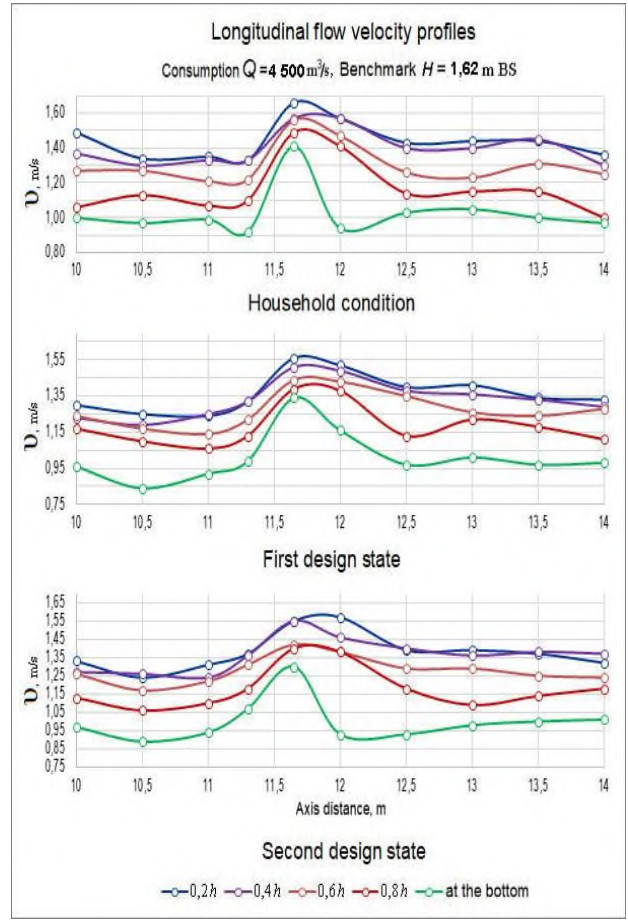

Fig. 8. Distribution of velocities along the ship's course.

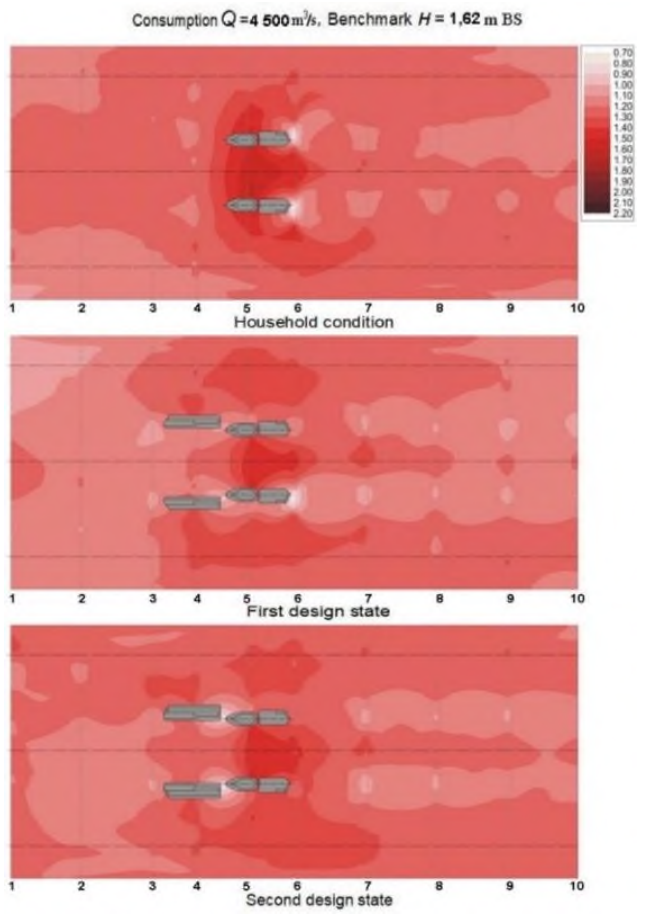

Fig. 9. Velocity field in the considered area for household (common) and design conditions at $Q$ $=4500 \mathrm{~m}^{3} / \mathrm{s}$ and $H=1.62 \mathrm{~m} \mathrm{BS}$. 


\section{Conclusions}

In general, based on the results of the analysis of laboratory research data on a hydraulic model, the following can be stated:

1. Installation of new structures in the form of supports of the designed bridge of the proposed geometry in the river channel does not cause significant changes in the flow structure. There is some redistribution of the flow rate in the site sections of the existing and designed bridges.

2. Average velocities in the navigable span of the existing bridge are slightly reduced when new supports are installed (from 1 to $7 \%$ ), the velocity diagram is aligned along the width of the central and side spans.

3. In the flow area behind the supports, a vortex wake is more clearly formed, which also distributes the flow along the channel width.

\section{References}

1. R.S. Chalov, Study of channels: theory, geography, practice. Volume 1 (LKI Publishing House Moscow, 2008)

2. R.S. Chalov, S.R. Chalov, Water Resources 47(3), 259-271 (2020) doi: 10.31857 / S0321059620030049

3. A.B. Klaven, Z.D. Kopaliani, Experimental Studies and Hydraulic Modeling of River Flows and Channel Processes (Nestor-Historiy, St. Petersburg, 2011)

4. G.L. Gladkov, R.S. Chalov, K.M. Berkovich, Gidromorfologiya rusel sudokhodnykh rek: Monografiya (2nd. Lan', St. Petersburg, 2019)

5. G.L. Gladkov, M.V. Zhuravlev, V.M. Katolikov, International Scientific Conference Energy Management of Municipal Facilities and Sustainable Energy Technologies EMMFT 2019. Advances in Intelligent Systems and Computing Springer, Cham https://doi.org/10.1007/978-3-030-57453-6_63

6. A.I. Aleksyuk, V.V. Belikov, N.M. Borisova, T.A. Fedorova, Water Resources 45(1), 11-17 (2018) doi: 10.1134/S0097807818050275

7. V.V. Belikov, N.M. Borisova, T.A. Fedorova, et al., Water Resources 46, S20-S28 (2019) https://doi.org/10.1134/S0097807819070029

8. A.N. Sukhodolov, V.I. Nikora, V.M. Katolikov, Journal of Hydraulic Research 49(3), 285-292 (2011) doi: 10.1080/00221686.2011.567760

9. G.L. Gladkov, M.V. Zhuravlev,Vestnik Gosudarstvennogo universiteta morskogo i rechnogo flota imeni admirala S.O. Makarova 11.6, 1044-1055 (2019) doi: 10.21821/2309-5180-2019-11-6-1044-1055

10. G.L. Gladkov, Vestnik Gosudarstvennogo universiteta morskogo i rechnogo flota imeni admirala S.O. Makarova 12.2, 336-346 (2020) doi: 10.21821/2309-5180-202012-2-336-346

11. G.L. Gladkov, K.P. Morgunov, Yu.K. Ivanovsky, Laboratory studies of the characteristics of the river flow in the area of the designed supports of the bridge crossing (Transport of the Russian Federation, SPb, 2020)

12. A.A. Voskoboinik, A.V. Voskoboinik, V.A. Voskoboinik, A.G. Marchenko, V.I. Nikishov, Applied Hydromechanics 8(3), 16-26 (2006) http://dspace.nbuv.gov.ua/handle/123456789/4760

13. L.C. Van Rijn, Ocean \& Coastal Management (2011) doi:10.1016/ j.ocecoaman.2011.05.004 
14. A.M. Kalyakin, E.V. Chesnokova, Magazine of Civil Engineering 3(55), 49-56 (2015) doi: 10.5862/MCE.55.6

15. A. Voskoboinick, V. Voskoboinick, V. Turick, O. Voskoboinyk, D. Cherny, L. Tereshchenko, Advances in Computer Science for Engineering and Education III. ICCSEEA 2020. Advances in Intelligent Systems and Computing, Springer, Cham (2021) https://doi.org/10.1007/978-3-030-55506-1_1

16. K.P. Morgunov, Gidravlika (Izdatel'stvo «Lan'», SPb, 2014)

17. M.A. Mikhalev, Fundamentals of physical modeling in fluid mechanics (2015) 